\title{
REFERRAL PATTERNS FOR CONSULTATION-LIAISON PSYCHIATRY AND THE UTILITY OF PRELIMINARY SCREENING: OBSERVATIONAL STUDY OF 448 OUTPATIENTS FROM A PORTUGUESE HOSPITAL
}

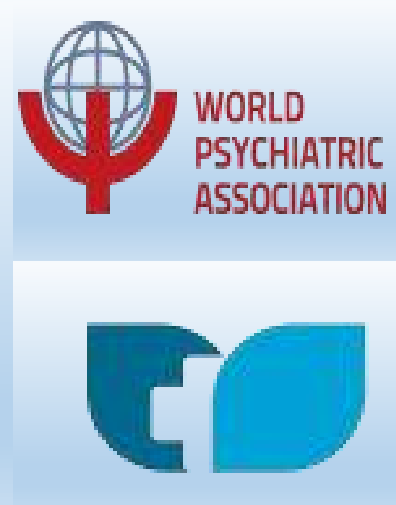

Nelson Couto*, Ana Teresa Pereira *, Lídia Sousa*, Diana Mota*, Luís Oliveira*, Lúcia Ribeiro*

* Medical Doctor, Psychiatry Department, Vila Nova Gaia/Espinho Hospital Center, Portugal

\section{OBJECTIVES}

To describe the demographic and clinical characteristics of consecutive referrals to a psychiatry liaison outpatient department, based within a Portuguese National Health Service general hospital, between May and December of 2018.

\section{BACKGROUND}

The increasing demand for psychiatric evaluations made by other medical specialties led the authors to try to implement a triage system. In this context, a consultation was organized to meet the needs of brief response and case selection.

\section{MATERIALS AND METHODS}

Patients were informed by telephone of the scheduling of their screening by the administrative team at least 1 month in advance. Over a 8-month period, information was recorded on the basis of non-identifiable clinical processes to save patients' privacy. These data, collected by 4 physicians, were later analyzed.

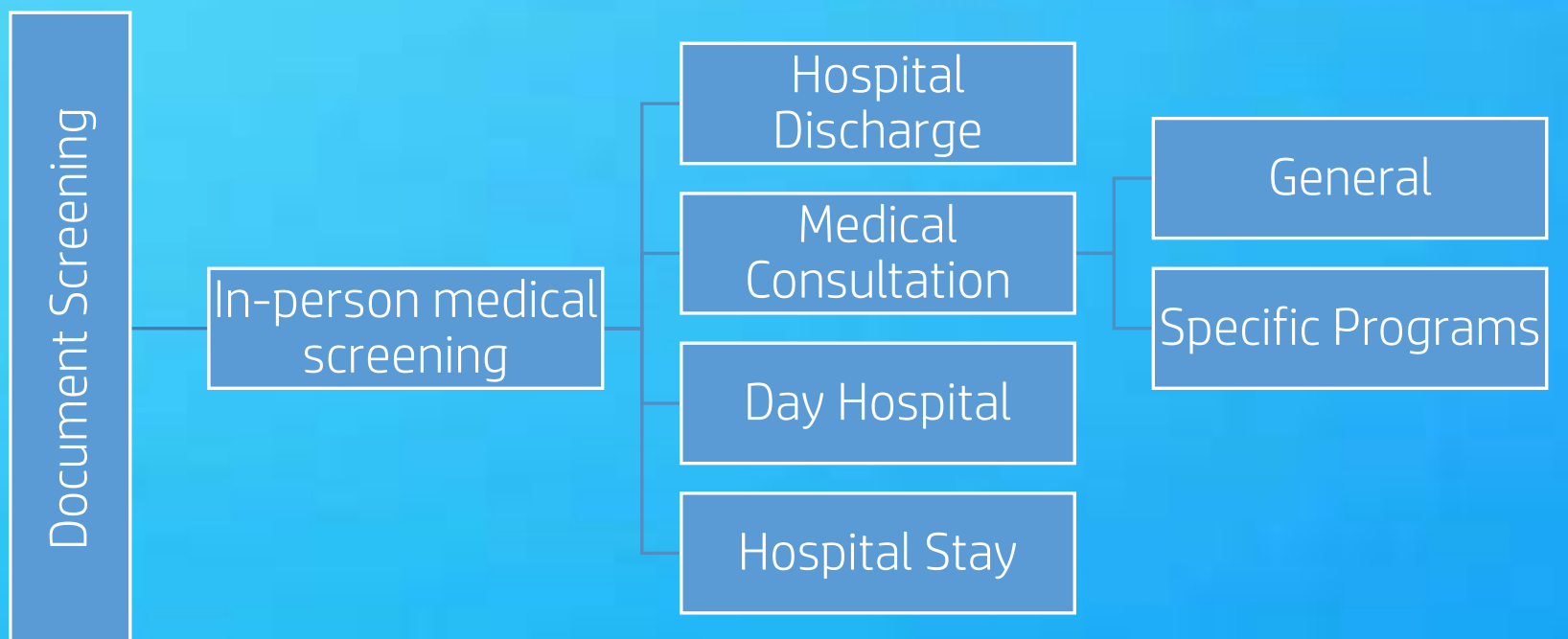

\section{RESULTS AND CONCLUSIONS}

\begin{tabular}{|c|c|c|}
\hline $\begin{array}{c}\text { Scheduled } \\
\text { Screening Queries }\end{array}$ & $\begin{array}{c}\text { Screening } \\
\text { Consultations }\end{array}$ & \% Performed \\
\hline 626 & 448 & 72 \\
\hline
\end{tabular}

626 Scheduled Screenings

Faults and Screening Effect

157 Triad Patients for Psychiatry (25\% of baseline)

\begin{tabular}{|l|l|l|l|}
\hline & $\begin{array}{l}\text { Total patients } \\
\text { observed }\end{array}$ & $\begin{array}{l}\text { Triad Patients } \\
\text { for Psychiatry }\end{array}$ & Variation (\%) \\
\hline $\begin{array}{l}\text { Absolute } \\
\text { number }\end{array}$ & 448 & 157 & -65 \\
\hline Middle Ages & 55,9 & 52,7 & $-5,8$ \\
\hline \% Women & 67 & 58 & $-13,5$ \\
\hline \% Employees & 39 & 34 & $-12,9$ \\
\hline $\begin{array}{l}\text { \% Prior } \\
\text { contact with } \\
\text { psychiatry }\end{array}$ & 31 & 35 & $+12,9$ \\
\hline $\begin{array}{l}\text { Most } \\
\text { referenced } \\
\text { medical } \\
\text { specialty }\end{array}$ & Neurology & Neurology & \\
\hline $\begin{array}{l}\text { Most prevalent } \\
\text { ICD-9 } \\
\text { diagnostic } \\
\text { group }\end{array}$ & $\begin{array}{l}\text { Neurotic } \\
\text { Disorders (300) }\end{array}$ & $\begin{array}{l}\text { Neurotic } \\
\text { Disorders (300) }\end{array}$ & \\
\hline
\end{tabular}

\section{Most common referencing pattern}

\begin{tabular}{|c|c|c|c|}
\hline Women & $\begin{array}{c}\text { Sixth } \\
\text { decade of } \\
\text { life }\end{array}$ & Jobless & $\begin{array}{c}\text { No prior } \\
\text { contact } \\
\text { with } \\
\text { psychiatry }\end{array}$ \\
\hline
\end{tabular}

